\title{
A Home Appliance Recognition System Using the Approach of Measuring Power Consumption and Power Factor on the Electrical Panel, Based on Energy Meter ICs ${ }^{*}$
}

\author{
Jefferson Z. Moro, Luís F. C. Duarte, Elnatan C. Ferreira, José A. Siqueira Dias \\ Department of Electronic and Microelectronic, State University of Campinas, Campinas, Brazil \\ Email: jeff@demic.fee.unicamp.br, lfduarte@demic.fee.unicamp.br, \\ elnatan@demic.fee.unicamp.br, siqueira@demic.fee.unicamp.br
}

Received February 16, 2013; revised March 17, 2013; accepted March 25, 2013

Copyright (C) 2013 Jefferson Z. Moro et al. This is an open access article distributed under the Creative Commons Attribution License, which permits unrestricted use, distribution, and reproduction in any medium, provided the original work is properly cited.

\begin{abstract}
Currently a large effort is being done with the intention to educate people about how much energy each electrical appliance uses in their houses, since this knowledge is the fundamental basis of energy efficiency programs that can be managed by the household owners. This paper presents a simple yet functional non-intrusive method for electric power measurement that can be applied in energy efficiency programs, in order to provide a better knowledge of the energy consumption of the appliances in a home.
\end{abstract}

Keywords: Energy Consumption; Energy Efficiency; Energy Metering; Power Measurement

\section{Introduction}

An accurate knowledge of the electric loads and appliance recognition is the foundation to promote energy efficiency, since it generates benefits both to the customers, who can manage the use of their appliances and obtain reduced costs in their electrical bills, and also for the utilities, which can optimize the operation and planning of the system [1].

Many techniques and methods have been used to measure the appliances power consumption and monitor their states. One approach to acquire the appliances power consumption and their states is to make use of a wireless sensor network. In this case, every appliance in a house must be connected to a smart sensor that performs the power measurement. The information of all the smart meters is then concatenated and is sent to system that generates a report $[2,3]$.

Another approach is to make use of a single intelligent power meter installed in the electric panel. This intelligent device monitors the power consumption of all appliances and then processes the monitored signal to identify the appliances based on load signatures. It minimizes the number of sensors needed to monitor all appliances and also reduces the complexity of the installation.

*This work was partially supported by CAPES.
Load Signature is an electrical expression that an appliance distinctly possesses regarding its electrical behaviour. It can be acquired from power consumption levels or from waveforms of electrical quantities such as voltage and current. Almost every electrical measurement can be treated as a load signature. It can be represented in the time domain [4], in the frequency domain [5] and can also be represented mathematically in terms of wavelets, eigenvalues, or components of the Singular Value Decomposition (SVD) [6].

In [7] the authors proposed a methodology of using load signatures and Genetic Algorithms (GA) to identify electrical appliances from a composite load signal. They introduced a classification method to group the appliances and how to disaggregate the composite load signals by a GA identification process which is generated from a random combination of load signatures from the distinct groups of appliances.

Recently a proposal for appliance recognition by measuring the power consumption of each circuit at the electrical panel distribution board has been presented [8]. The technique uses a sophisticated meter and based on the instantaneous measurements results of the energy meter and on expected behaviour of the residents of the house provides good results for the appliance recognition process.

In this paper we propose the use of a similar approach 
of measuring the electrical circuits at circuit level, with one power meter for each circuit breaker, but using a low cost hardware. The proposed hardware is composed of simple energy meter integrated circuits and a recognition algorithm which does not rely on an expected behaviour of the residents, since this can change drastically form culture to culture.

\section{Objective}

The goal of this work is the development of a system able to measure and separate the power consumption of different appliances in a house, in order to provide better knowledge of the energy consumption of the appliances in a residence.

\section{Hardware}

The developed hardware has four modules: a PCB with the energy meter IC, a microcontroller that is responsible for the management of all modules, a memory for data storage and a Wi-Fi module which transmits the data to any device with Wi-Fi connection. A block diagram of the system is shown in Figure 1.

The power meter is basically composed of a modular printed circuit board with small current transformers, one for each circuit breaker at the distribution panel. The voltage at circuit is also fed to modular board, so that each energy meter integrated circuit (one per module) receives both the current (via current transformer) and the voltage at that circuit. The energy meter IC is the ADE7763 [9]. This IC was chosen because it has the capability of measuring both active and apparent energy, and it communicates to others ICs using SPI. Thus, only a few wires are required to interconnect all the modular energy meter boards with the microcontroller.

The microcontroller is an ATmega328. A high-performance Atmel 8-bit AVR RISC-based microcontroller with $32 \mathrm{kB}$ of flash memory, $2 \mathrm{kB}$ of RAM and $1 \mathrm{kB}$ of EEPROM. It is an inexpensive and easy to programs IC with many open source codes and libraries available.

The Wi-Fi network was chosen in order to allow the energy information data to be accessed both from computers and mobile devices with wireless internet capability, such as tablets and smartphones.

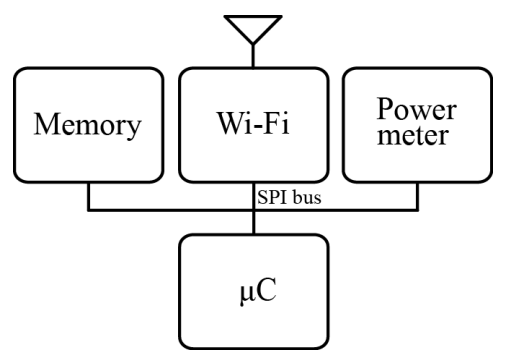

Figure 1. Block diagram of the proposed system.

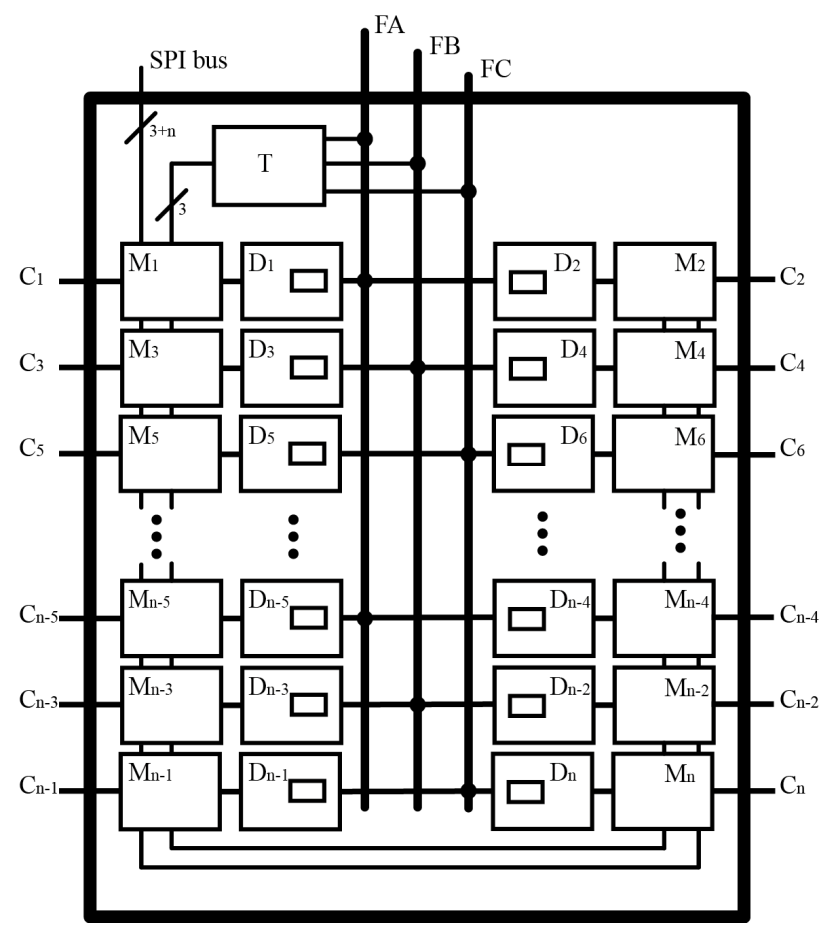

Figure 2. Diagram of installation showing the measurement performed in each circuit apart.

On Figure 2, T represents a group of three potential transformers supplied by each one of the three phases of the mains line. These transformers reduce the voltage and also isolate the grid allowing the ICs to sample the voltage. The current of each electric circuit $C_{i}$ that comes from circuit breakers $D_{i}$, pass through a current transformer and is then acquired by the a dedicated power meter IC $\mathrm{M}_{\mathrm{i}}$.

The prototype was mounted on the form of two boards: one main board and one measuring board. The main board is composed by the memory, the Wi-Fi module, the processing unit (microcontroller), power supply, potential transformers and auxiliary circuits. Figures 3 and 4 show the schematic of the main board circuit and a photograph of the assembled main board is shown in Figure 5.

The measuring board is basically composed by the measuring electronics circuits and by the currents transformers. It was designed and fabricated according to the schematic diagram presented in Figure 6.

Each measuring board was fabricated with nine IC power meters. The position of the current transformer was set-up to be perfectly aligned with the wire input of the circuit breaker, so that the installation of the board is extremely simple. The complete measuring board with the current transformers soldered on it is presented in Figure 7.

The final assembling of the measurement board in an electrical panel is presented in Figure 8. As it can be 


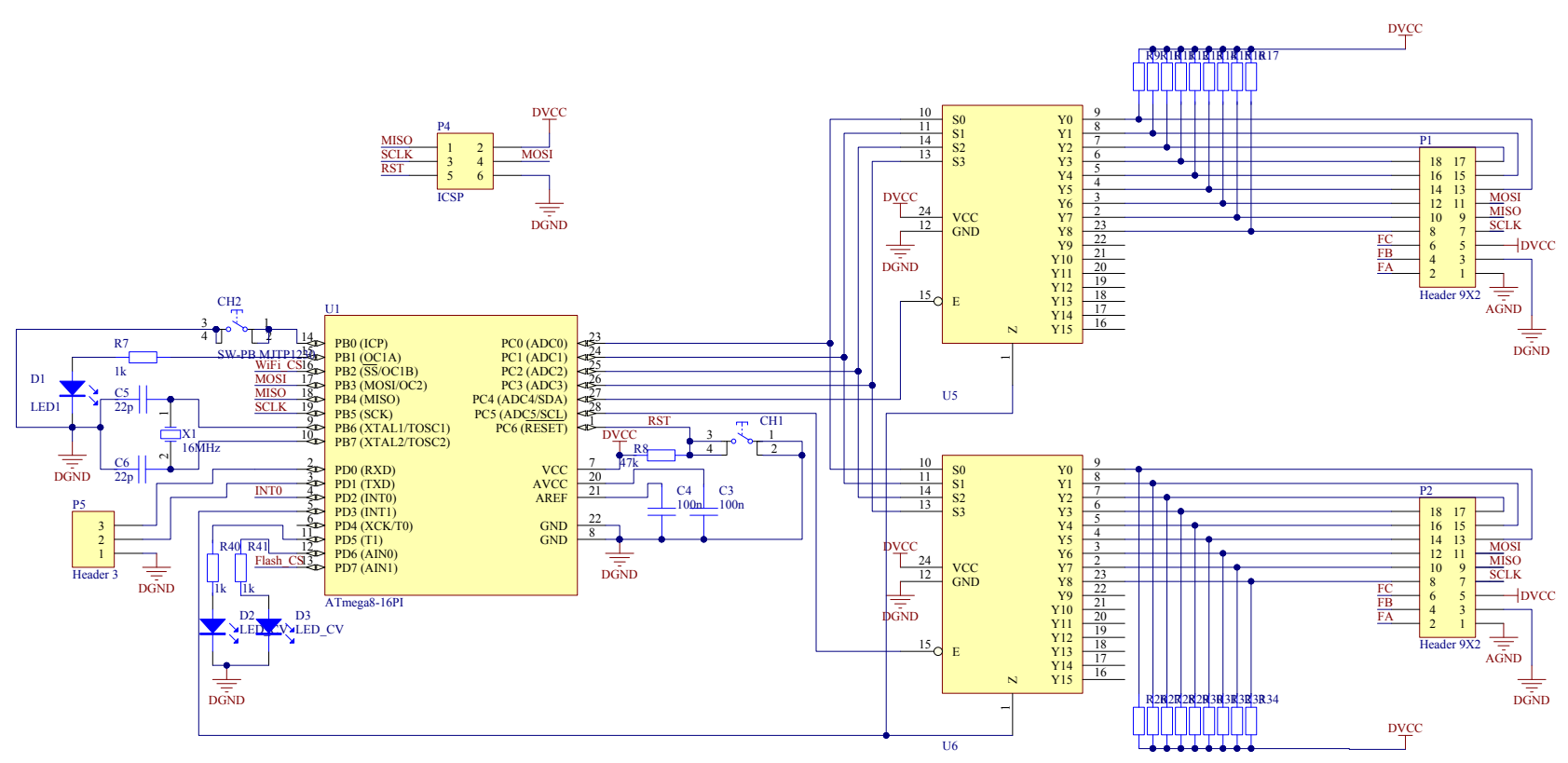

Figure 3. Schematic of the main board circuit including the $\mu \mathrm{C}$ and the switching connections.

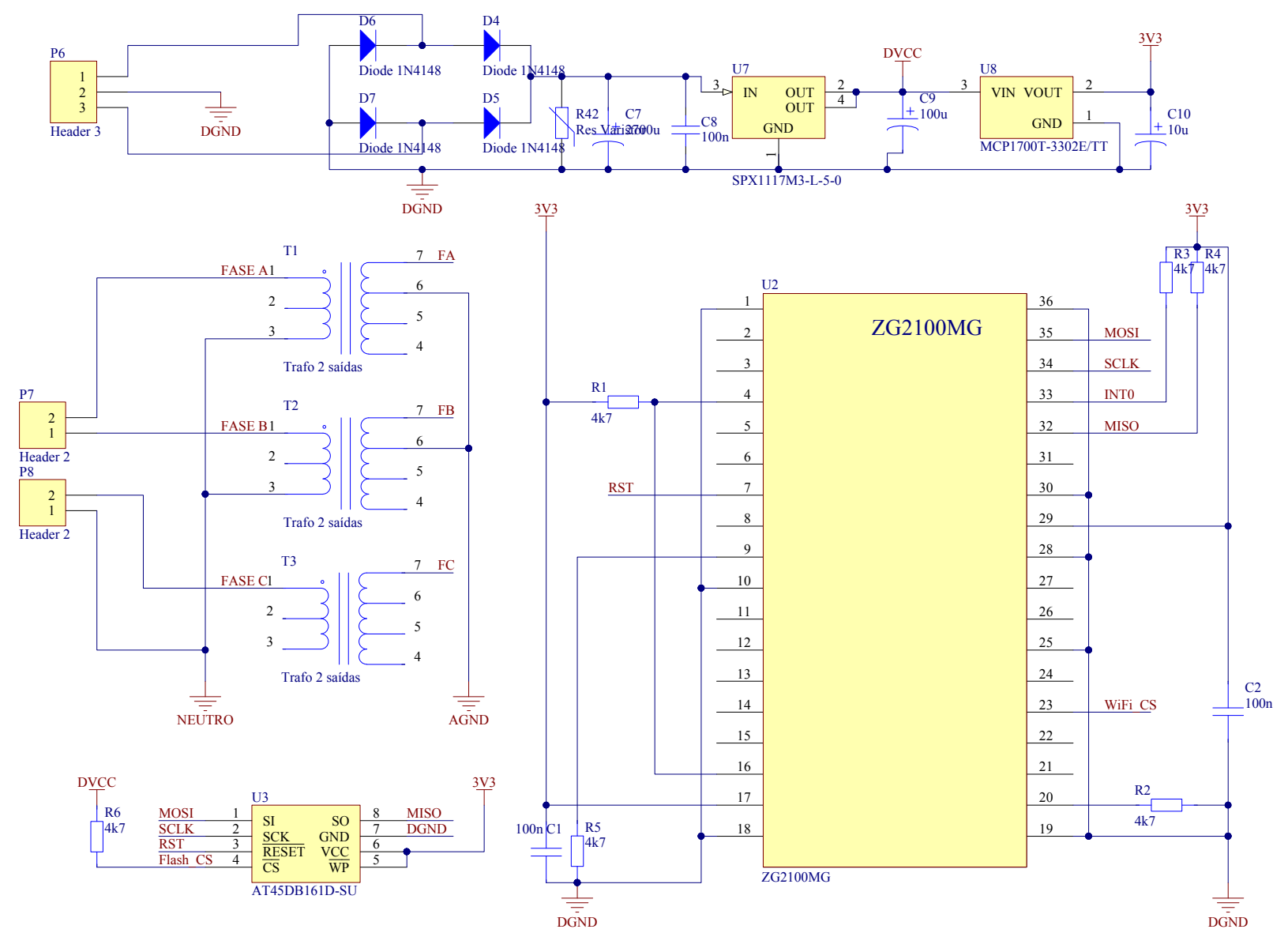

Figure 4. Schematic of the main board circuit including the Wi-Fi module and the power sources.

observed, the board is on the background at the panel and, except for the current transformer, it can hardly be no- ticed. Since the board receives 3 phases and the neutral, the voltage of the corresponding phase is selected with 


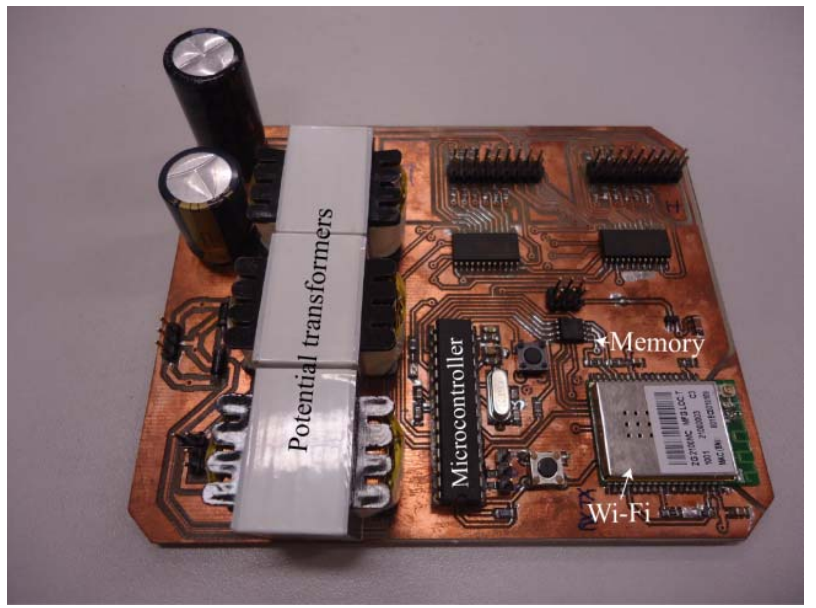

Figure 5. Main board.

configuration jumpers existent in each input of every measuring circuit.

Each one of the electric circuits can be referred to six voltage values: three possible phase voltages Van, Vbn and Vcn that are respectively the three voltage of the three phases $\mathrm{A}, \mathrm{B}$ and $\mathrm{C}$, referenced to neutral, and three line voltage $\mathrm{VAB}, \mathrm{VBC}$ and $\mathrm{VCA}$, that are the voltage of the three phases referenced between each other. The notation follows the following rule: VXY $=\mathrm{Vxn}-\mathrm{Vyn}$. Using jumpers it is possible to select any voltage signals to each energy power meter, properly setting up the system according to the circuit each meter is connected to. Figure 9 shows two examples illustrating this situation. Figure 10 the phase selector jumpers.

\section{Software}

The firmware installed in the microcontroller program memory is detailed in the flowchart in Figure 11. The main task executed on the initialization sets up the power meters ICs, performing an individual circuit calibration. After that, the firmware verifies if the user wants to make actualizations on memory data. If yes, the program is switched to memory loading routine.

The power meter ICs store the measured data. Once per minute, the microcontroller reads the data of each circuit via Serial Peripheral Interface (SPI) and then writes the data in the external flash memory.

The data in the external flash memory is available to the user via an embedded web server that is accessed via Wi-Fi. After reading the power meters, the microcontroller verifies if there is any request from the Wi-Fi module to access the web page. If so, the request is treated by the TCP/IP stack, and the cycle restarts. Otherwise the cycle is restarted immediately.

The load recognition software was developed using JavaScript. It was stored in the external flash memory. From the moment that the web page is requested by the user, the JavaScript code is sent to client browser and
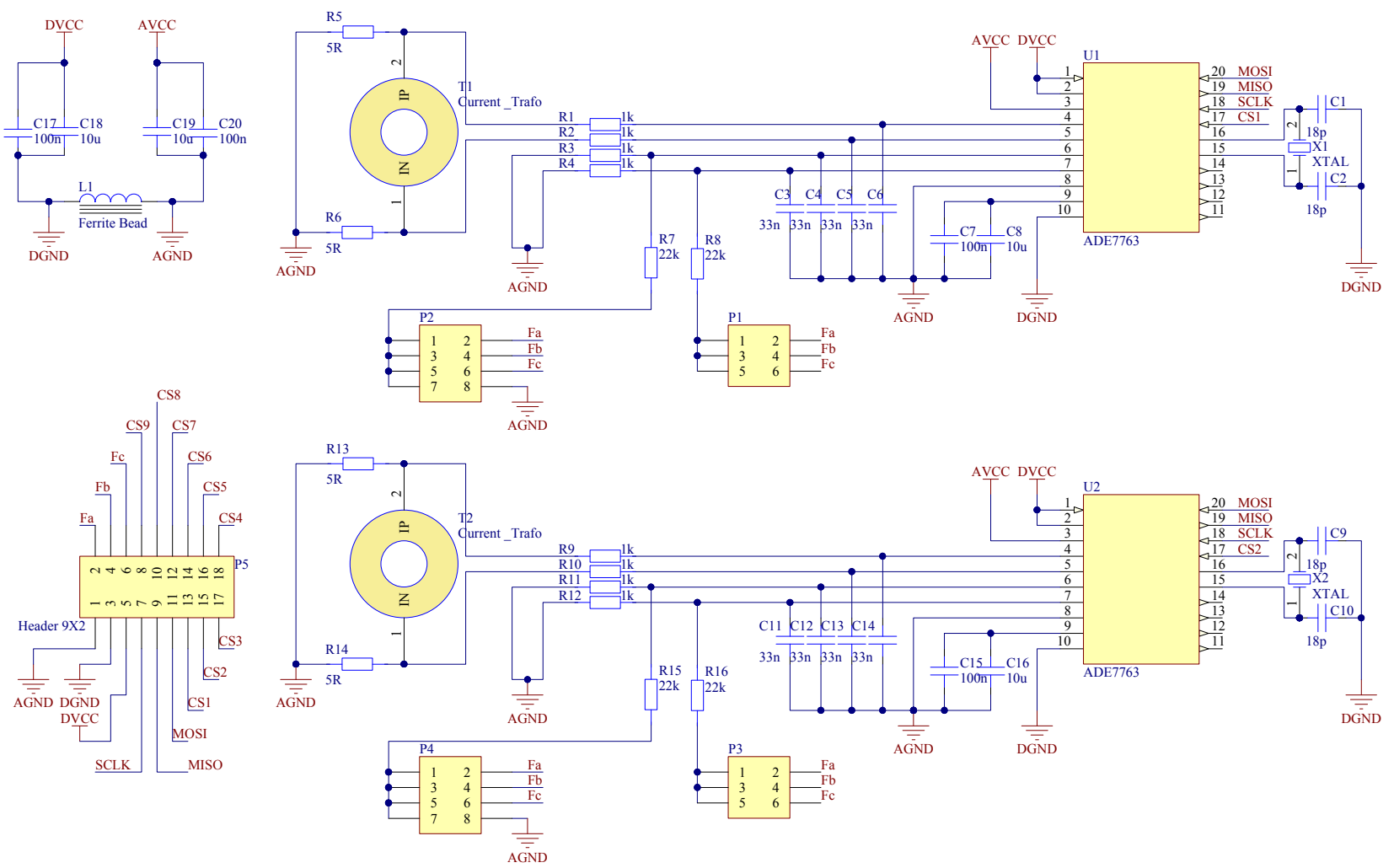

Figure 6. Schematic of the measuring board circuit. 


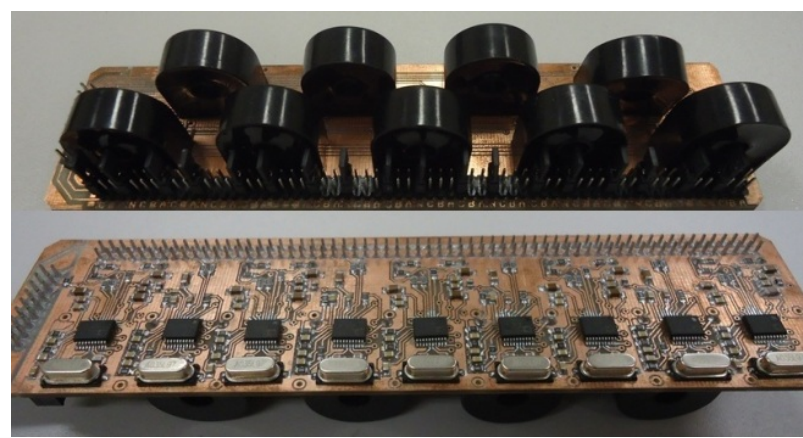

Figure 7. Measuring board.

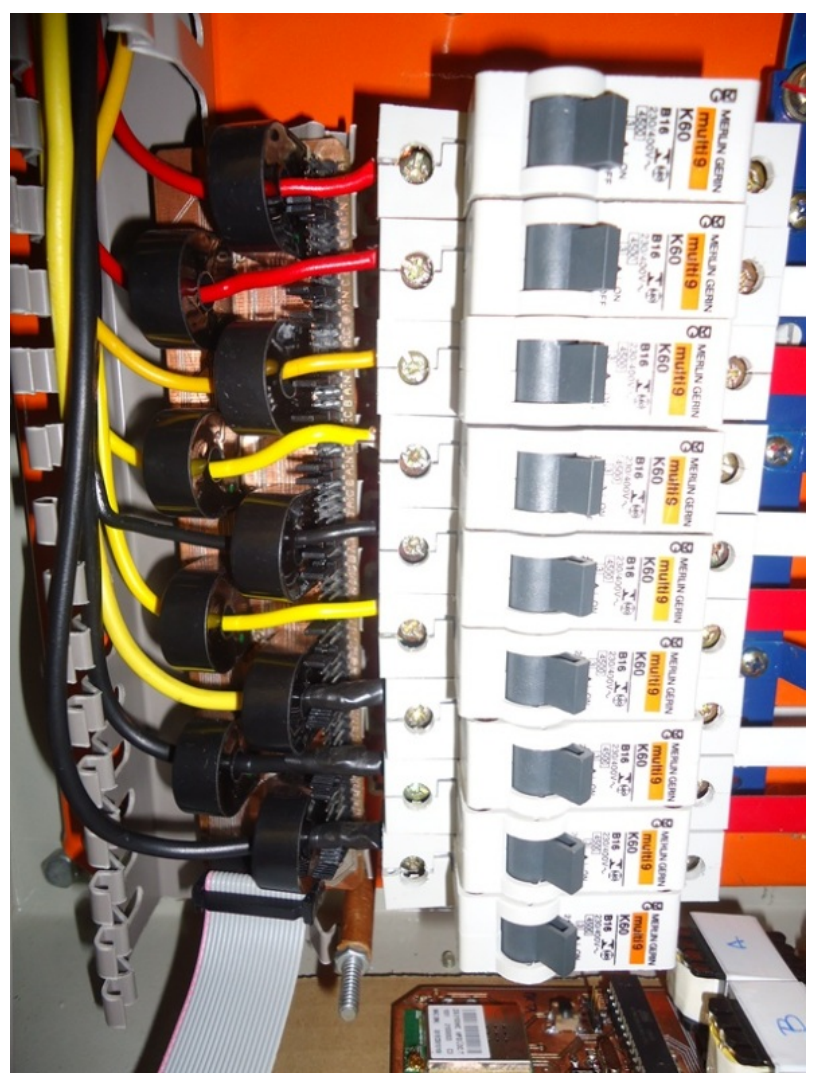

Figure 8. Measuring board installed inside of the circuit breakers box.

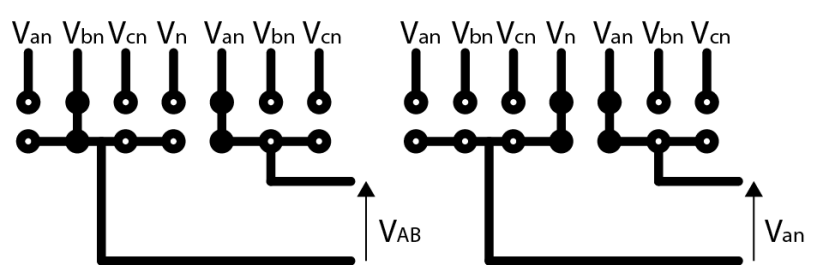

Figure 9. Example of phase voltage and line voltage, selected by jumpers.

there, it is executed by client computer. This is a way to reduce the work of the microcontroller. Figure 12 shows the flowchart of the program that is sent to browser of the user through the Wi-Fi module.

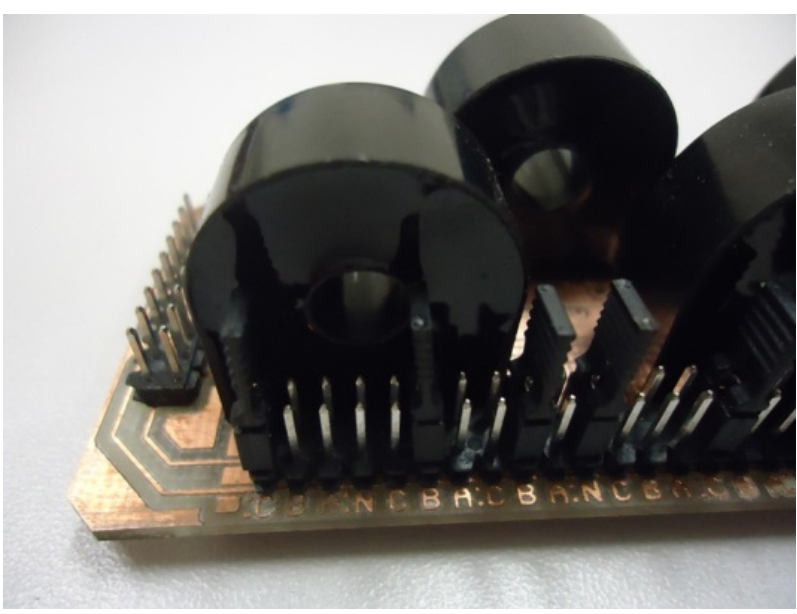

Figure 10. Detail of the measuring board, showing the selectors jumpers.

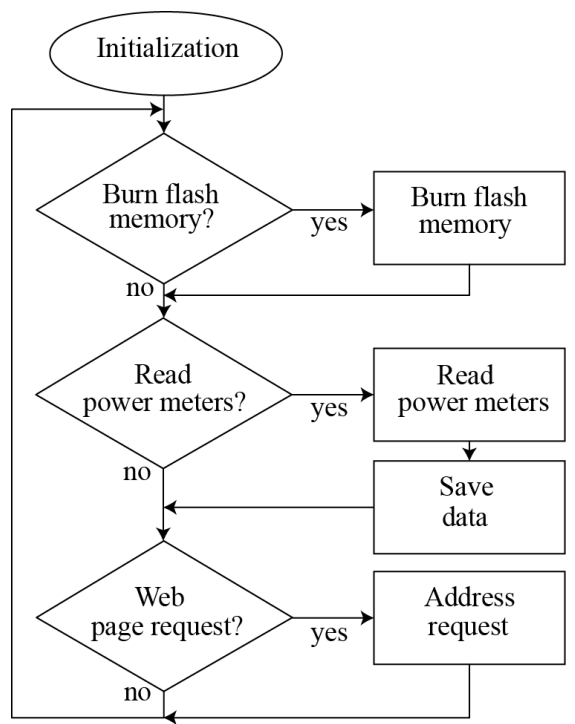

Figure 11. Flowchart of the embedded firmware.

\section{System Setup and Operation}

After being installed, the system is initiated in learning mode, with all the electrical appliances of a given circuit turned off. Next, each appliance is turned on and its name and location can be entered with the use of a notebook or any other device with internet connection.

The active and reactive energy are measured during a small period of time (typically $15 \mathrm{~s}$ ) and the value of active energy and the power factor of that is sent to the PC. Since the measurement is made during a known period of time, the PC calculates the active power and the power factor of the appliance and stores it in a table, associating this data with the location and the type of load. For example, the table will store the data: Dining room, ceiling lamp, 100 watts, P.F. 0.98.

In the sequence this load is turned off and one by one all the other appliances which are connected to the same 


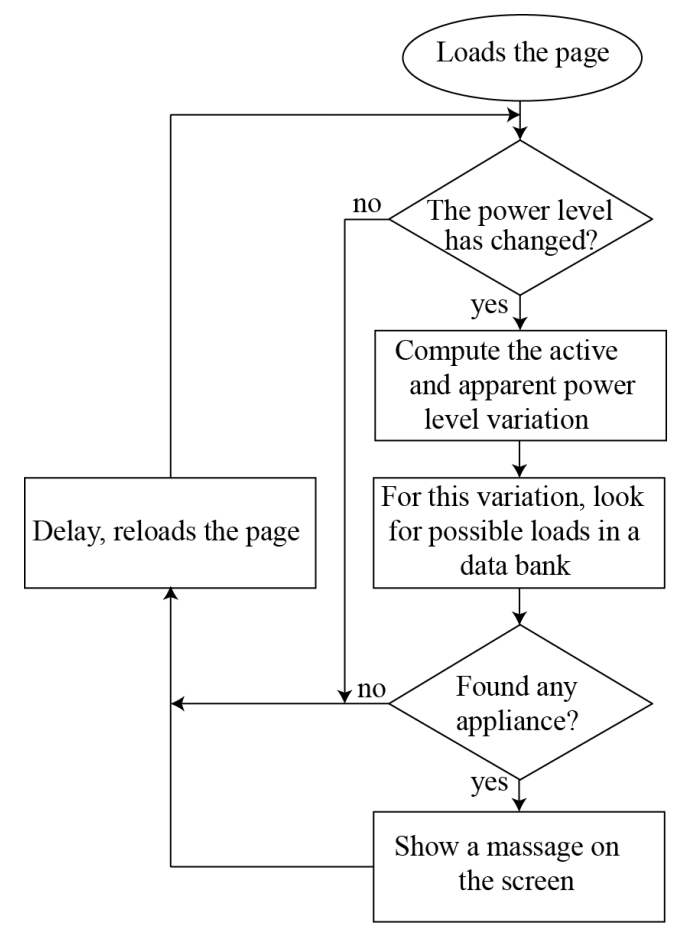

Figure 12. Flowchart of the program executed by the browser.

circuit breaker are turned on, identified and stored by the software.

The same procedure is made for every circuit until the whole house is completely identified. If an appliance is used in more than one place, and these locations are not protected by the same circuit breaker, the load can be registered in both locations without problems. As for example, if a coffee machine is used in the kitchen and in the dining room.

If an appliance is substituted (for example, the bedroom incandescent lamps) the system has to be updated by deleting the old information about the replaced devices and acquiring the new one.

Once every appliance signature is recorded, the system starts to operate, measuring each circuit separately. Then, in reduced universe, steps in power consumption are monitored. Not only the active power steps are monitored, but also the apparent power steps. It allows the microcontroller to calculate the power factor and then use it together with the active power step as a load signature.

For example, if in a given house there are 30 lamps of 15 watts distributed in 10 rooms (10 different circuits) the identification software will have to "guess" only between 3 lamps instead of 30 .

This technique reduces significantly the number of appliances that have to be identified, making the correct identification much easier.

Furthermore, the use of simple energy meter ICs that can measure both active and reactive power leads to an identification of load appliances that is much more pow- erful than the other techniques currently available.

\section{Experimental Results}

The system was tested and the loads were chosen to intentionally create difficulty to the proper identification of appliances in the systems that do not measure reactive energy.

A test set-up was prepared with one $40 \mathrm{~W}$ incandescent lamp, one $20 \mathrm{~W}$ compact fluorescent lamp and one $20 \mathrm{~W}$ incandescent lamp. The system was capable of identifying when the $40 \mathrm{~W}$ lamp was on by the energy consumption (measuring during $1 \mathrm{~s}$ ) and also could identify precisely which $20 \mathrm{~W}$ lamp was on, because of the P.F.

A second test was made turning on/off the $40 \mathrm{~W}$ incandescent lamp and the two $20 \mathrm{~W}$ lamps simultaneously, in order to simulate a $40 \mathrm{~W}$ appliance. The software can detect that a step of $40 \mathrm{~W}$ was measured with a P.F. which is not in the table. So, it combines all possible loads that result in a power step of $40 \mathrm{~W}$ and calculates the P.F. to determine which combination matches the measured value.

Using this technique it was possible to detect properly all combinations of these 3 appliances.

\section{Conclusions}

This paper has presented a novel technique of "per circuit" electrical power metering system able to identify loads.

The "per circuit" measurement technique significantly reduces the computational cost of the project, while it increases the chance of recognizing the loads correctly.

It also facilitates the insertion of a new load in the system by allowing the user to switch off only the appliances in the same circuit and not all appliances in the house during the learning event.

Investing in a hardware a little more elaborated, allied to a good distribution of the currents transducers in the circuit breakers box, have shown that the load identification becomes easier, so the program used to do that identification can be executed by a simple 8 bits microcontroller, which parallel executes others tasks such as those requested by TCP/IP stack.

\section{REFERENCES}

[1] L. F. C. Duarte, E. C. Ferreira and J. A. S. Dias, "Energy Measurement Techniques for Energy Efficiency Programs," In: Moustafa Eissa, Ed., Energy Efficiency-The Innovative Ways for Smart Energy, the Future Towards Modern Utilities, InTech, 2012, pp. 193-208. doi:10.5772/47791

[2] H. Serra, J. Correia, A. J. Gano, A. M. de Campos and I. Teixeira, "Domestic Power Consumption Measurement and Automatic Home Appliance Detection," Proceedings of 
2005 IEEE International Workshop Intelligent Signal Processing, 1-3 September 2005, pp. 128-132.

[3] L. F. C. Duarte, J. D. Zambianco, D. Airoldi, E. C. Ferreira and J. A. S. Dias, "Characterization and Breakdown of the Electricity Bill Using Custom Smart Meters: A Tool for Energy-Efficiency Programs," International Journal of Circuits, Systems and Signal Processing, Vol. 5, No. 2, 2011, pp. 116-123.

[4] C. Laughman, K. Lee, R. Cox, S. Shaw, S. Leeb, L. Norford and P. Armstrong, "Power Signature Analysis," IEEE Power and Energy Magazine, Vol. 1, No. 2, 2003, pp. 5663. doi:10.1109/MPAE.2003.1192027

[5] J. Duan, D. Czarkowski and Z. Zabar, "Neural Network Approach for Estimation of Load Composition," IEEE International Symposium on Circuit and Systems (ISCAS), New York, 23-26 May 2004, pp. 988-991.

[6] W. K. Lee, G. S. K. Fung, H. Y. Lam and F. H. Y. Chan,
"Exploration on Load Signatures," Proceedings of International Conference on Electrical Engineering (ICEE), 2004.

http://lucente.us/db/pubs/ICEE2004Signature-reprint.pdf

[7] J. S. K. Leung, K. S. H. Ng and J. W. M. Cheng, "Identifying Appliances Using Load Signatures and Genetic Algorithms," Proceedings of International Conference on Electrical Engineering (ICEE), 2007. http://www.icee-con.org/papers/2007/Oral_Poster\%20Pa pers/01/ICEE-031.pdf

[8] G. Lin, S. Lee, J. Y. Hsu and W. Jih, "Applying Power Meters for Appliance Recognition on the Electric Panel," Proceedings of the 5th IEEE Conference on Industrial Electronics and Applications (ICIEA), Taichung, 15-17 June 2010, pp. 2254-2259.

[9] Analog Devices, "ADE7763 Data Sheet," Single-Phase Active and Apparent Energy Metering IC, 2009. 